\title{
Impact of selection for uterine capacity on the placental transcriptome
}

\author{
B.A. Freking', J.R. Miles', S.R. Bischoff'2, S. Tsai' ${ }^{2}$, N. Hardison'², Y. Xia ${ }^{3}$, \\ D.J. Nonneman', J.L. Vallet' and J.A. Piedrahita ${ }^{2}$ \\ 'USDA, ARS, U.S. Meat Animal Research Center, Clay Center, NE USA; ${ }^{2}$ Department of Molecular \\ Biomedical Sciences, College of Veterinary Medicine, North Carolina State University, Raleigh, NC \\ USA; ${ }^{3}$ Center for Biotechnology, University of Nebraska, Lincoln, NE USA
}

Direct single trait selection for 11 generations resulted in a 1.6 pig advantage for uterine capacity (UC) while average birth and placental weights at term remained unchanged. Uterine capacity was defined as the total number of fully-formed pigs produced to term when ovulation rate was not limiting, using a unilateral hysterectomy-ovariectomy model. A serial slaughter experiment conducted throughout gestation determined the critical time period for the line difference in litter size was already established between d 25 and 45 of gestation and generated direct evidence of differential relative growth rates for placental tissues at these times. Timing of line differences in fetal survival as well as anecdotal evidence of tissue structural differences pointed to the developing placental tissue as a target of particular interest. Our objective was to gain insight into placental transcriptional changes during this critical stage of gestation and identify genetic loci impacted by quantitative selection for uterine capacity.

Thirty gilts each from the UC and control (CO) lines were subjected to unilateral hysterectomyovariectomy at approximately $160 \mathrm{~d}$ of age and mated within line at approximately $280 \mathrm{~d}$. Gilts were slaughtered at $d 25,30$, or 40 of gestation. Fetal and placental tissues were obtained from each live embryo. Fetal liver samples were used to extract DNA and determine sex of each fetus by PCR. Two male and two female embryos closest to the litter mean for placental weight were chosen to represent each litter sampled $n=3$ litters per line and time point combination). Placental tissues were pooled within litter and total RNA was extracted. Samples were labeled and hybridized to Affymetrix porcine array chips $(n=18)$ using the manufacturers suggested protocols. Signal intensities were normalized using GC content Robust Multi-array Average (GCRMA) on the probe level data. Filtering was based on perfect match intensities as implemented for Affymetrix Human arrays. Two-way ANOVA (two lines and three stages) was performed. Threshold values were set at a minimum of 1.5 fold difference and the false discovery rate was set to $P<0.05$ (Benjamini and Hochberg algorithm). Less stringent two-way comparisons (t-tests) were also conducted between lines within each gestation stage. GeneSifter ${ }^{\mathscr{D}}$ software web tools were utilized to conduct the analyses and generate the gene lists.

An additional analysis was conducted to examine the potential for a bioinformatics method of identifying single feature polymorphism (SNP) targets between the two lines associated with the behavior of the expression data. A gene by gene linear mixed model of probe intensity variation from 11 probes per gene target was investigated to identify line $x$ probe interactions. Log2-transformed perfect-match intensities for all observations were fit to a linear mixed model that broadly corrected for effects of breed and array. A gene-specific mixed model was fit to the normalized intensities (residuals from first model) accounting for fixed breed, probe, and breed-by-probe interaction effects, and a random array effect. Probes that produced statistical evidence (qvalue $\leq 0.05$ to account for multiple testing and $\mid$ fold change $\mid \geq 2$ ) for the breedby-probe interaction were identified as candidates containing SNP. The interaction was declared 
significant when Prob $t<0.0064$ and would indicate a putative coding region polymorphism associated with line within that probe location. Validation of this approach was conducted in a previous experiment comparing Meishan and occidental breed placental tissues with an $87 \%$ success rate using the same threshold values of $q<0.05$ for false discovery rate and with greater than 2-fold difference in estimated corrected probe-by-breed interaction effects. The great majority of these single feature polymorphisms are discovered in the middle portions of the 25-mer probe targets.

A total of 4171 targets on the array exceeded $P$ value and threshold limits for the main effect of stage (2230 up-regulated and 1839 down-regulated from d 25 to $\mathrm{d} 40$ ). Two targets, LIM domain and actin-binding protein 1 (LIMA1) and a hypothetical protein LOC51524 (transmembrane protein 138) approached highly stringent thresholds for significance of the main effect of line ( $P$ $<0.08$ ) in the two-way ANOVA with both targets up-regulated in the UC line and increasing during these stages of gestation. Expression data for both loci were validated as differentially expressed by QPCR $(P<0.05)$ and also increased with gestation age. The function of LIMA1 is to bind to actin monomers and filaments, increase the number and size of actin stress fibers and inhibit membrane ruffling. It inhibits actin filament depolymerization, promotes bundling of actin filaments, delays filament nucleation and reduces formation of branched filaments. Both genes could be predicted to play roles in cell migration, suggesting participation in placental folded bilayer formation.

Less stringent analyses of two-way comparisons within each time point indicated increased expression occurred more frequently than decreased expression in the UC line. The number of up-regulated targets relative to the CO line identified totaled 219, 407, and 234 for gestation stages d 25,30, and 40, respectively. In contrast, the number of down-regulated targets relative to the $\mathrm{CO}$ line identified totaled 36,71 , and 47 for gestation stages $\mathrm{d} 25,30$, and 40 , respectively. This overall expression profile is consistent with observations reached previously, that these lines differed in relative growth patterns of fetal and placental tissues. Genes of interest identified in these lists revealed the importance of pathways such as integrin signaling pathway (calpains, collagen, fibronectin) and lipid metabolism (adiponectin receptors).

Analysis of probe $x$ line data yielded a large number (5617) of putative transcribed SNP between the lines. Merging these data with the expression information indicated an overlap of 762 targets where day effects were also significantly different in the two-way ANOVA, and an overlap of 179 targets from the pair-wise contrasts within each stage. Therefore this overlapping list of targets offers evidence of both expression differences in the placenta as well as genetic variation between the lines that could be contributing to genetic variation for uterine capacity. Both lines originated from the same four-breed composite genetic base prior to the 11 generations of selection applied. Due to the small sampling of data generated from the microarrays, it is also likely a large number of these putative line-specific polymorphisms are associated with effects of genetic drift. However, these targets offer an intriguing start to directly untangling the impact of genetic selection for uterine capacity. It is clear that a more thorough investigation by cluster and pathway or network analysis will also require better annotation of targets on the array. The experimental and analytical approaches in this study identified both expression and polymorphism differences to pursue for identification of genetic markers contributing to genetic variation in uterine capacity from a unique germplasm resource. 